\title{
Four-dimensional flow magnetic resonance imaging: Beyond beautiful pictures!
}

\author{
Jonathan W. Weinsaft, MD, ${ }^{a}$ Mario Gaudino, MD, ${ }^{b}$ and Leonard N. Girardi, MD
}

\author{
From the Departments of ${ }^{\mathrm{a}}$ Medicine (Cardiology) and ${ }^{\mathrm{b}}$ Cardiothoracic Surgery, Weill Cornell Medicine, New \\ York, NY. \\ Disclosures: Authors have nothing to disclose with regard to commercial support \\ Received for publication June 22, 2018; revisions received June 22, 2018; accepted for publication June 25, 2018 \\ available ahead of print Aug 7, 2018 \\ Address for reprints: Leonard N. Girardi, MD, O. Wayne Isom Professor of Cardiothoracic Surgery, Department \\ of Cardiothoracic Surgery, New York-Presbyterian Weill Cornell Medicine, 525 E 68th St, New York, NY \\ 10065 (E-mail: lngirard@med.cornell.edu). \\ J Thorac Cardiovasc Surg 2019;157:477-8 \\ $0022-5223 / \$ 36.00$ \\ Copyright (c) 2018 by The American Association for Thoracic Surgery \\ https://doi.org/10.1016/j.jtcvs.2018.06.075
}

Cardiothoracic surgical advances have paralleled progress in cardiovascular imaging. Coronary bypass surgery followed the introduction of coronary angiography, mitral valve repair was facilitated by developments in echocardiography, and aortic surgery has been refined by highresolution anatomic data yielded by tomographic methods. Despite substantial advances, challenges remainincluding the need for more precise algorithms to stratify preoperative risk, guide surgical timing, and optimize postprocedural surveillance. Further imaging developments hold the potential to address these key needs, with the ultimate goal of improved clinical outcomes for surgical patients.

Time-resolved phase-contrast magnetic resonance imaging (MRI) with 3-directional velocity encoding and volumetric coverage, or 4-dimensional (4D) flow, constitutes a new imaging advance with extraordinary potential. The technique can quantify flow throughout volumetric regions of interest, enabling physiologic assessment throughout the thoracic aorta, a marked expansion from 2-dimensional imaging. Four-dimensional flow captures vascular anatomy and time-resolved velocity field of blood flow, enabling quantitative assessment of vessel geometry, calculation of velocity, and the analysis of complex flow patterns and hemodynamic derivatives, including vorticity, pressure gradients, wall shear stress, and kinetic energy. Four-dimensional flow can be acquired with high spatial resolution, enabling flow to be assessed in small, irregularly contoured structures. Recent MRI advances have reduced scan time (10-15 minutes), facilitating clinical application of this technique.

In this issue of The Journal of Thoracic and Cardiovascular Surgery, de Beaufort and colleagues ${ }^{1}$ report on the reproducibility of $4 \mathrm{D}$ flow $\mathrm{MRI}$ in an ex vivo model of aortic dissection and in patient data. Findings demonstrate similarly high intraobserver and interobserver correlations for ex vivo and clinical data ( $r$ of $0.96-0.98$ ), paralleled

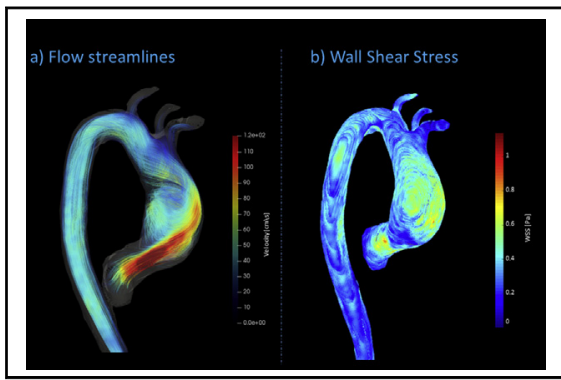

Four-dimensional flow magnetic resonance imaging of the thoracic aorta.

\section{Central Message}

Four-dimensional flow magnetic resonance imaging has great clinical potential in aortic surgery.

See Article page 467.

by small mean differences. Notably, 4D flow consistently demonstrated higher flow volumes in the ascending aorta than did 2-dimensional flow. Methodologic differences were greater in patients with repaired type I (vs type III) dissections; the reasons for this are uncertain. This important study expands on previous 4D flow work, including investigations examining aneurysm pathogenesis in bicuspid aortic valves ${ }^{2}$ and research by our group, which used 4D flow to address physiologic consequences of recreation of the sinuses of Valsalva during valve-sparing surgery. ${ }^{3}$ The topic of aortic dissection, as addressed by de Beaufort and colleagues, ${ }^{1}$ represents one in which current surgical treatment indications are based on nonspecific symptoms or anatomic criteria that can be imprecise with respect to longitudinal risk for aortic growth and dissection expansion.

Despite immense advances in surgical technologies during the past decades, decision making is often based on static imaging data. Limits of this approach are clear. For example, aortic size is a cornerstone for aneurysm repair, despite the fact that a substantial proportion of patients with genetically mediated aortic aneurysms have dissection occur before the aneurysm has reached the size-based criteria for surgery. ${ }^{4}$

In the current era of precision medicine, 4D flow MRI holds the potential to characterize novel sequelae of aortic pathologies and thus inform physiologically tailored surgical decision making. The current study of de Beaufort and colleagues, ${ }^{1}$ provides key validation of this powerful 
technique, justifying the need for further studies to test the incremental utility of 4D flow with respect to guidance of interventional strategies and prediction of surgical outcomes.

\section{References}

1. de Beaufort HW, Shah DJ, Patel AP, Jackson MS, Spinelli D, Yang EY, et al Four-dimensional flow cardiovascular magnetic resonance in aortic dissection: assessment in an ex vivo model and preliminary clinical experience. J Thorac Cardiovasc Surg. 2019;157:467-76.e1.
2. Rodriguez-Palomares JF, Dux-Santoy L, Guala A, Kale R, Maldonado G, Teixidó-Turà G, et al. Aortic flow patterns and wall shear stress maps by 4Dflow cardiovascular magnetic resonance in the assessment of aortic dilatation in bicuspid aortic valve disease. J Cardiovasc Magn Reson. 2018;20:28.

3. Galea N, Piatti F, Sturla F, Weinsaft JW, Lau C, Chirichilli I, et al. Cornell international consortium for aortic surgery (CICAS). Novel insights by $4 \mathrm{D}$ flow imaging on aortic flow physiology after valve-sparing root replacement with or without neosinuses. Interact Cardiovasc Thorac Surg. 2018;26:957-64.

4. Weinsaft JW, Devereux RB, Preiss LR, Feher A, Roman MJ, Basson CT, et al; GENTAC Registry Investigators. Aortic dissection in patients with genetically mediated aneurysms: incidence and predictors in the GenTAC registry. J Am Coll Cardiol. 2016;67:2744-54. 